\title{
Long Chains of Nearly Doubled Primes
}

\author{
By Günter Löh
}

\begin{abstract}
A chain of nearly doubled primes is an ordered set $\left\{a_{1}, a_{2}, \ldots, a_{\lambda}\right\}$ of prime numbers, interlinked by $a_{k}=2 a_{k-1} \pm 1$. A search for long chains of this kind has been performed in the range $a_{1}<2^{50}$. Chains of length up to 13 have been found. Shorter chains have been counted in some restricted ranges. Some of these counts are compared with the frequencies predicted by a quantitative version of the prime $k$-tuples conjecture.
\end{abstract}

1. Introduction. Let us consider a sequence of nearly doubled integers, recursively defined by $a_{k}=2 a_{k-1}+\varepsilon, \varepsilon \in\{-1,+1\}$, starting with some $a_{1}$. If $a_{1}$ is a prime, one can ask how many consecutive primes occur in the sequence. We say that the ordered set $\left\{a_{1}, a_{2}, \ldots, a_{\lambda}\right\}$ forms a prime chain of length $\lambda$, if $a_{\lambda+1}$ is the first composite number of the sequence. The value $\varepsilon$ has to be kept fixed throughout the whole prime chain, because a change of sign for $a_{1}>3$ would yield the numbers $a_{1}, a_{2}=2 a_{1}+\varepsilon, a_{3}=2\left(2 a_{1}+\varepsilon\right)-\varepsilon$, one of which is divisible by 3 . A well-known example for the case $\varepsilon=+1$ is the prime chain $\{89,179,359,719,1439,2879\}$ of length $\lambda=6$.

Guy [3, pp. 12-13] pointed out that prime chains appear as a special case when commonly used methods for proving that a number $p$ is prime are applied. These methods require the factorization of either $p-1$ or $p+1$. If $p-\varepsilon=2 q$, where $q$ is another prime, the size of the problem is only reduced by one bit. This worst case may occur several times in sequence.

In the case $\varepsilon=+1, \lambda \geq 2$, the prime $a_{1}$ is a so-called Sophie Germain prime. For these primes $a_{1}$, where $a_{1}>2$, the first case of Fermat's last theorem holds (Ribenboim [8, pp. 55-56]).

We can express $a_{k}$ as $a_{k}=2^{k-1} a_{1}+\varepsilon\left(2^{k-1}-1\right)$. According to Fermat's little theorem, if $k=a_{1}>2$, then $a_{1}$ divides $a_{k}$. This means that for $a_{1}>2$ the length $\lambda$ of a prime chain cannot exceed $a_{1}-1$ (Nederpelt et al. [7]). Actually, most of the prime chains are considerably shorter. It is not known whether arbitrarily long chains exist.

Extending Yates' notation [13, p. 115], we describe the set of primes constituting a prime chain by $S_{\varepsilon}\left(\lambda, a_{1}\right)$. Thus, for fixed $\varepsilon$, each prime $a_{1}$ uniquely determines a chain $S_{\varepsilon}\left(\lambda, a_{1}\right)$ which contains $\lambda-1$ shorter chains $S_{\varepsilon}\left(\lambda+1-k, a_{k}\right), k=2,3, \ldots, \lambda$, whenever $\lambda>1$.

We say that a prime chain $S_{\varepsilon}\left(\lambda, a_{1}\right)$ is complete if it cannot be extended downwards, that is, if the rational number $a_{0}=\left(a_{1}-\varepsilon\right) / 2$ is a composite integer or

Received January 6, 1987; revised August 18, 1988.

1980 Mathematics Subject Classification (1985 Revision). Primary 11B37; Secondary 11A41, $11-04$.

Key words and phrases. Nearly doubled primes, prime chains, Cunningham chains, prime $k$ tuples conjecture. 
smaller than 2. Hence, for a given $\varepsilon$, each prime belongs to one and only one complete chain.

It seems reasonable to consider pairs of mutually related prime chains, both of which grow simultaneously. But for most of the possible configurations, one of the chains breaks down prematurely. For instance, looking at the chains $S_{-1}\left(\lambda_{-1}, a_{1}\right)$ and $S_{+1}\left(\lambda_{+1}, a_{1}\right)$, we observe that for $a_{1}>3$ either $\lambda_{-1}$ or $\lambda_{+1}$ must be 1 , since one of the numbers $2 a_{1}-1,2 a_{1}+1$ must be divisible by 3 . On the other hand, we may consider pairs of chains $S_{+1}\left(\lambda_{+1}, r-1\right)$ and $S_{-1}\left(\lambda_{-1}, r+1\right)$, where $(r-1, r+1)$ is a pair of twin primes. In this case, the length of neither chain is restricted in such a way. Moreover, it is not known how many consecutive twin prime pairs formed by both chains can occur.

For $\varepsilon=+1$, the first remarkable prime chains were presented by Cunningham [2] in 1907. Accordingly, the chains $S_{+1}\left(\lambda, a_{1}\right)$ are also called Cunningham chains. He gave the above example $S_{+1}(6,89)$ as well as $S_{+1}(6,63419)$ and $S_{+1}(6,127139)$. The length $\lambda=6$ was not surpassed for more than half a century.

In 1965 Lehmer [6] found several chains of length $\lambda=7$ for both values of $\varepsilon$, specifically, $S_{+1}(7,1122659), S_{+1}(7,2164229), S_{+1}(7,2329469), S_{+1}(7,10257809)$, $S_{+1}(7,10309889)$, and, for $\varepsilon=-1$, the chains $S_{-1}(7,16651)$, and $S_{-1}(7,165901)$. One additional chain in his paper, with $\varepsilon=-1$ and alleged length $\lambda=7$ starting with $a_{1}=67651$, must be ruled out since $a_{5}=1082401=601 \cdot 1801$. Lehmer also gave some hints on how to efficiently compute such chains. He expected that chains of length $\lambda=8$ should begin to appear for $a_{1} \approx 10^{9}$.

Fifteen years later, the first chains of length $\lambda=8$ for both $\varepsilon$ were published by Lalout and Meeus. In [5] they reported that Nelson discovered $S_{+1}(8,19099919)$ and Lalout discovered $S_{-1}(8,15514861)$. Furthermore, all complete chains of length $\lambda \geq 5$ with $a_{1}<5 \cdot 10^{5}$, and length $\lambda \geq 6$ with $a_{1}<2 \cdot 10^{7}$ were given in [5]. In an editorial note by Nelson to that paper, it was stated that chains of length $\lambda=9$ do exist for both $\varepsilon$, but only $S_{-1}(9,857095381)$ was explicitly given. It was also reported that for $a_{1}<2 \cdot 10^{9}$ no chains of length $\lambda=10$ had been found. It should be mentioned that Sumiyama [11] first published the chain $S_{+1}(9,85864769)$ already known to Nelson.

In this paper, we have extended the search for long chains as far as $a_{1}<2^{50} \approx$ $1.12 \cdot 10^{15}$. We have succeeded in finding several prime chains of length $\lambda, 10 \leq \lambda \leq$ 12 for both values of $\varepsilon$, and one of length $\lambda=13$ in the case $\varepsilon=-1$. Moreover, for all $\lambda$ we determined the frequencies of complete chains up to $a_{1}<2^{26}$. For some larger limits up to $a_{1}<2^{36}$, short chains were discarded in order to reduce the amount of computation. The programs were coded in FORTRAN 77 and ran for about 520 CPU hours on a SIEMENS 7.882 computer.

2. Computations. In all our computations we used powers of 2 as search limits, for a reason to be indicated later. Basically, only complete chains starting with a given prime $a_{1}$ were determined. For $a_{1}<2^{26}$ we examined all primes as possible first members of a complete chain. For $a_{1}>2^{26}$, however, we chose some minimal length $l$ and restricted ourselves to prime chains with length $\lambda \geq l$. When doing so, one can establish congruence conditions for $a_{1}$ involving the first $\mu$ primes in order to speed up the search. Such conditions were already explicitly given by Cunningham [2] for $l=4, \mu=4$ and by Eisner [7] for $l=7, \mu=7$. 
For instance, if we only look for chains of minimal length $l=12$, in the case $\varepsilon=-1$ the number $a_{1}$ must satisfy the following congruences for the first $\mu=9$ primes:

$$
\begin{aligned}
& a_{1} \equiv 1(\bmod 2 \cdot 3 \cdot 5 \cdot 11 \cdot 13) \\
& a_{1} \equiv 1,2,3, \text { or } 5(\bmod 7) \\
& a_{1} \equiv 1,4,6,7,8,11,12,13, \text { or } 15(\bmod 17) \\
& a_{1} \equiv 1,4,7,12,13,16, \text { or } 18(\bmod 19) \\
& a_{1} \equiv 1,2,3,4,5,7,9,10,13,14,17, \text { or } 19(\bmod 23) .
\end{aligned}
$$

Let $q_{j}$ denote the $j$ th prime. For $j \geq 2$, let $r_{j}$ be the least positive power of 2 with $2^{r_{j}} \equiv 1\left(\bmod q_{j}\right)$, and set $r_{1}=1$. Then only $\prod_{j=1}^{\mu}\left(q_{j}-\min \left(r_{j}, l\right)\right)$ numbers out of a full cycle of $\prod_{j=1}^{\mu} q_{j}$ numbers remain for further testing. So in the above example, we have a cycle length of $2 \cdot 3 \cdot 5 \cdot 7 \cdot 11 \cdot 13 \cdot 17 \cdot 19 \cdot 23=223092870$ numbers, of which only $1 \cdot 1 \cdot 1 \cdot 4 \cdot 1 \cdot 1 \cdot 9 \cdot 7 \cdot 12=3024$ have to be considered as a possible $a_{1}$.

On these remaining numbers we performed trial division using the primes $q_{\mu+1}$, $q_{\mu+2}, \ldots, q_{m}$, where $m$ had to be chosen appropriately. The single remainder $a_{1} \bmod q_{j}$ suffices to determine whether any one of the $a_{k}, k \leq l$, is divisible by $q_{j}$. The surviving numbers $a_{1}$ and the subsequent members of a possible chain were tested for compositeness by evaluating $13^{a_{k}-1} \bmod a_{k}$, for $k=1,2,3, \ldots$, until the first composite number was encountered. Thus, at least $l$ probable primes in succession had to be gathered to obtain a chain in the range being explored. Once such a chain was found, all probable primes were validated by conventional methods. The choice of $m=300$ brought the total computational effort for trial division and compositeness testing close to a minimum.

We searched for chains of length $\lambda \geq l, l=4,5,6$ and $a_{1}<2^{29}, 2^{33}, 2^{36}$, respectively. In these regions we found many prime chains of length $\lambda$ up to 9 for both $\varepsilon$. In addition, for $\varepsilon=+1$ two chains of length $\lambda=10$ were found. Looking for longer chains, we continued the search with $l=10$ and $a_{1}<2^{43}$. In this region we found several chains; the first members of these are listed in Table 1 . It should be noted that in the case $\varepsilon=+1$ a chain of length $\lambda=12$ was found before a chain of length $\lambda=11$ had occurred. Furthermore, in Table 2 the prime chains with $\lambda \geq l=12$ and $a_{1}<2^{50}$ are presented.

For the reader's convenience we have compiled Table 3, which shows the first appearance of a complete chain for each $\varepsilon$ and all $\lambda$ up to 12 resp. 13. The chains of length $\lambda \geq 10$ are taken from the previous Tables 1 and 2 .

Now, let $(r-1, r+1)$ be a pair of twin primes and consider the two chains $S_{+1}\left(\lambda_{+1}, r-1\right)$ and $S_{-1}\left(\lambda_{-1}, r+1\right)$. We ask for the maximal number $\lambda=$ $\min \left(\lambda_{-1}, \lambda_{+1}\right)$ of consecutive twin prime pairs produced by both chains. For the first appearance of such a twin chain of length $\lambda$, we observed the following values of $r: \lambda=1, r=4 ; \lambda=2, r=6 ; \lambda=3, r=211050 ; \lambda=4, r=253680 ; \lambda=5$, $r=41887255410 ; \lambda=6, r=73768891456260$.

Turning to the frequencies of shorter chains, in Tables 4 and 5 we give the counts $c_{\varepsilon}\left(\lambda, 2^{\nu}\right)$ of all complete prime chains of length $\lambda$ for $a_{1}<2^{\nu}, 16 \leq \nu \leq 36$, where the table values are restricted to the regions indicated above. The corresponding counts $d_{\varepsilon}\left(\lambda, 2^{\nu}\right)$ of all prime chains, including those which are not complete, can be derived from the values $c_{\varepsilon}\left(\lambda, 2^{\nu}\right)$, and vice versa. 
TABLE 1

Complete chains with $a_{1}<2^{43}$ and length $\lambda \geq 10$

\begin{tabular}{rcrc}
$\varepsilon=+1$ & \multicolumn{1}{c}{$\varepsilon=-1$} \\
\hline$a_{1}$ & $\lambda$ & $a_{1}$ & $\lambda$ \\
\hline 26089808579 & 10 & 205528443121 & 10 \\
65639153579 & 10 & 207526905301 & 10 \\
372339715439 & 10 & 237441500011 & 10 \\
551652886619 & 10 & 428822526001 & 10 \\
554688278429 & 12 & 605727738331 & 10 \\
570901515029 & 10 & 629912195221 & 10 \\
624758088239 & 10 & 1220127683941 & 10 \\
665043081119 & 11 & 1389122693971 & 11 \\
721438465439 & 10 & 2339669157541 & 11 \\
1243541923469 & 10 & 2514864555061 & 10 \\
1341807015119 & 10 & 2605339529101 & 10 \\
2027840556719 & 10 & 3264821602351 & 10 \\
2452499778299 & 10 & 3689229625951 & 10 \\
2486906850119 & 10 & 4704099859471 & 10 \\
2834226458909 & 10 & 4723155667231 & 10 \\
3495773443139 & 10 & 5321563702621 & 10 \\
3717568843559 & 10 & 6131306463151 & 10 \\
3919801172429 & 10 & 6289424815411 & 10 \\
4228633787069 & 10 & 6454116380581 & 10 \\
4315740820379 & 10 & 6860403225391 & 10 \\
4389398045009 & 10 & 7165183292851 & 11 \\
4709436287819 & 10 & 7557661146751 & 10 \\
5213499728579 & 11 & 7603857496951 & 10 \\
5496251895419 & 10 & 7697789715331 & 10 \\
6424044070289 & 11 & 7918316060821 & 10 \\
7623279613949 & 10 & & \\
\hline & & &
\end{tabular}

TABLE 2

Complete chains with $a_{1}<2^{50}$ and length $\lambda \geq 12$

\begin{tabular}{rccc}
\multicolumn{1}{c}{$\varepsilon=+1$} & \multicolumn{2}{c}{$\varepsilon=-1$} \\
\hline \multicolumn{1}{c}{$a_{1}$} & $\lambda$ & $a_{1}$ & $\lambda$ \\
\hline 554688278429 & 12 & 216857744866621 & 12 \\
17287267757759 & 12 & 758083947856951 & 13 \\
175469208383039 & 12 & 890299395628501 & 12 \\
485385457835849 & 12 & 952373350504291 & 12 \\
\hline
\end{tabular}

This interdependence between a table of $c_{\varepsilon}\left(\lambda, 2^{\nu}\right)$ and a similar table of $d_{\varepsilon}\left(\lambda, 2^{\nu}\right)$ strongly relies on the fact that the limits for $a_{1}$ are given as powers of 2 . For this implies that a chain starting with an odd prime $a_{1}$ in the interval $2^{\nu-1}<a_{1}<2^{\nu}$ has its successive members $a_{k}$ in the intervals $2^{\nu+k-2}<a_{k}<2^{\nu+k-1}, k \geq 1$. This can easily be seen to be true for both values of $\varepsilon$, when the closed form expression for $a_{k}$ given in the introduction is recalled. 
TABLE 3

The smallest $a_{1}$ starting a complete chain of length $\lambda$

\begin{tabular}{rrr} 
& $\varepsilon=+1$ & $\varepsilon=-1$ \\
\hline$\lambda$ & $a_{1}$ & $a_{1}$ \\
\hline 1 & 13 & 11 \\
2 & 3 & 7 \\
3 & 41 & 2 \\
4 & 509 & 2131 \\
5 & 2 & 1531 \\
6 & 89 & 385591 \\
7 & 1122659 & 16651 \\
8 & 19099919 & 15514861 \\
9 & 85864769 & 857095381 \\
10 & 26089808579 & 205528443121 \\
11 & 665043081119 & 1389122693971 \\
12 & 554688278429 & 216857744866621 \\
13 & $?$ & 758083947856951 \\
\hline
\end{tabular}

TABle 4

Counts of complete chains $c_{\varepsilon}\left(\lambda, 2^{\nu}\right)$ with $a_{1}<2^{\nu}$ and length $\lambda$

$$
\varepsilon=+1
$$

\begin{tabular}{rrrrrrrrrrr}
\hline$\nu$ & $\lambda=1$ & 2 & 3 & 4 & 5 & 6 & 7 & 8 & 9 & 10 \\
\hline 16 & 5326 & 608 & 107 & 21 & 4 & 2 & 0 & 0 & 0 & 0 \\
17 & 10109 & 1077 & 192 & 31 & 5 & 3 & 0 & 0 & 0 & 0 \\
18 & 19242 & 1887 & 346 & 49 & 9 & 3 & 0 & 0 & 0 & 0 \\
19 & 36795 & 3387 & 564 & 71 & 14 & 4 & 0 & 0 & 0 & 0 \\
20 & 70205 & 6185 & 999 & 113 & 25 & 5 & 0 & 0 & 0 & 0 \\
21 & 134307 & 11297 & 1711 & 196 & 37 & 11 & 1 & 0 & 0 & 0 \\
22 & 257193 & 20816 & 3029 & 325 & 64 & 18 & 3 & 0 & 0 & 0 \\
23 & 493482 & 38295 & 5331 & 531 & 110 & 36 & 3 & 0 & 0 & 0 \\
24 & 948147 & 71045 & 9516 & 904 & 176 & 51 & 8 & 0 & 0 & 0 \\
25 & 1825113 & 131502 & 16999 & 1522 & 289 & 77 & 11 & 1 & 0 & 0 \\
26 & 3518641 & 243887 & 30189 & 2643 & 493 & 105 & 16 & 2 & 0 & 0 \\
27 & & & & 4542 & 846 & 159 & 21 & 2 & 1 & 0 \\
28 & & & & 8009 & 1397 & 261 & 39 & 3 & 2 & 0 \\
29 & & & & 14039 & 2342 & 410 & 66 & 5 & 3 & 0 \\
30 & & & & & 4032 & 681 & 91 & 8 & 3 & 0 \\
31 & & & & & 6965 & 1102 & 157 & 14 & 3 & 0 \\
32 & & & & & 12023 & 1849 & 254 & 22 & 4 & 0 \\
33 & & & & & 20653 & 3075 & 445 & 38 & 6 & 0 \\
34 & & & & & & 5244 & 694 & 72 & 10 & 0 \\
35 & & & & & & 8821 & 1084 & 118 & 12 & 1 \\
36 & & & & & & 14970 & 1766 & 185 & 17 & 2 \\
\hline
\end{tabular}

The values $d_{\varepsilon}\left(\lambda, 2^{\nu}\right)$ can be derived from those of $c_{\varepsilon}\left(\lambda, 2^{\nu}\right)$ by summation over certain diagonals using the formula

$$
d_{\varepsilon}\left(\lambda, 2^{\nu}\right)=\sum_{n=0}^{\nu-16} c_{\varepsilon}\left(\lambda+n, 2^{\nu-n}\right)+d_{\varepsilon}\left(\lambda+\nu-15,2^{15}\right) .
$$


TABLE 5

Counts of complete chains $c_{\varepsilon}\left(\lambda, 2^{\nu}\right)$ with $a_{1}<2^{\nu}$ and length $\lambda$

$\varepsilon=-1$

\begin{tabular}{rrrrrrrrrrr}
\hline$\nu$ & $\lambda=1$ & 2 & 3 & 4 & 5 & 6 & 7 & 8 & 9 & 10 \\
\hline 16 & 5313 & 595 & 124 & 18 & 5 & 0 & 1 & 0 & 0 & 0 \\
17 & 10131 & 1039 & 208 & 25 & 7 & 0 & 1 & 0 & 0 & 0 \\
18 & 19282 & 1854 & 365 & 37 & 14 & 0 & 2 & 0 & 0 & 0 \\
19 & 36816 & 3359 & 587 & 58 & 24 & 3 & 2 & 0 & 0 & 0 \\
20 & 70183 & 6206 & 1008 & 92 & 39 & 4 & 2 & 0 & 0 & 0 \\
21 & 134167 & 11364 & 1757 & 164 & 61 & 7 & 3 & 0 & 0 & 0 \\
22 & 257131 & 20734 & 3070 & 302 & 84 & 16 & 4 & 0 & 0 & 0 \\
23 & 493390 & 38284 & 5445 & 520 & 126 & 22 & 5 & 0 & 0 & 0 \\
24 & 947949 & 71033 & 9621 & 867 & 187 & 40 & 8 & 1 & 0 & 0 \\
25 & 1825061 & 131520 & 16880 & 1484 & 311 & 60 & 12 & 1 & 0 & 0 \\
26 & 3518643 & 244165 & 29930 & 2651 & 523 & 96 & 18 & 2 & 0 & 0 \\
27 & & & & 4572 & 888 & 149 & 25 & 2 & 0 & 0 \\
28 & & & & 7999 & 1469 & 239 & 42 & 2 & 0 & 0 \\
29 & & & & 13935 & 2452 & 382 & 64 & 2 & 0 & 0 \\
30 & & & & & 4177 & 618 & 99 & 9 & 1 & 0 \\
31 & & & & & 7079 & 1055 & 165 & 14 & 1 & 0 \\
32 & & & & & 12108 & 1791 & 250 & 24 & 2 & 0 \\
33 & & & & & 21143 & 2977 & 403 & 34 & 5 & 0 \\
34 & & & & & & 5043 & 667 & 54 & 9 & 0 \\
35 & & & & & & 8635 & 1105 & 85 & 14 & 0 \\
36 & & & & & & 14787 & 1739 & 152 & 20 & 0 \\
\hline
\end{tabular}

TABLE 6

Number of chains with $a_{1}<2^{\nu}$ and minimal length $l$

$\varepsilon=+1 \quad \varepsilon=-1$

\begin{tabular}{rcrrrrr}
\hline$l$ & $\nu$ & Approx. & Count & Ratio & Count & Ratio \\
\hline 2 & 20 & 8583 & 8051 & 0.938 & 8088 & 0.942 \\
& 23 & 50477 & 48024 & 0.951 & 48165 & 0.954 \\
& 26 & 309743 & 297544 & 0.961 & 297412 & 0.960 \\
\hline 3 & 20 & 1499 & 1246 & 0.831 & 1250 & 0.834 \\
& 23 & 7474 & 6475 & 0.866 & 6603 & 0.883 \\
& 26 & 39981 & 35625 & 0.891 & 35355 & 0.884 \\
\hline 4 & 20 & 252 & 164 & 0.651 & 169 & 0.671 \\
& 23 & 1016 & 777 & 0.765 & 789 & 0.777 \\
& 26 & 4665 & 3699 & 0.793 & 3728 & 0.799 \\
& 29 & 23178 & 18769 & 0.810 & 18784 & 0.810 \\
\hline
\end{tabular}

The last term of this sum, with first argument $\lambda+\nu-15 \geq 2$, must be supplied additionally. The required values of $d_{\varepsilon}\left(\lambda, 2^{15}\right)$ are as follows: $d_{+1}\left(\lambda, 2^{15}\right)=$ $382,70,19,2,1$ for $\lambda=2,3,4,5,6$, respectively, and $d_{-1}\left(\lambda, 2^{15}\right)=389,79,14,3,0,1$ for $\lambda=2,3,4,5,6,7$, respectively. For larger values of $\lambda$, all $d_{\varepsilon}\left(\lambda, 2^{15}\right)$ are zero. 
Since every prime $p$ starts one (not necessarily complete) prime chain with $a_{1}=$ $p$, we have, for fixed $\nu$,

$$
\sum_{\lambda \geq 1} d_{+1}\left(\lambda, 2^{\nu}\right)=\sum_{\lambda \geq 1} d_{-1}\left(\lambda, 2^{\nu}\right)=\pi\left(2^{\nu}\right)
$$

where $\pi(n)$ is the number of all primes not exceeding $n$. As a check of the tabulated values $c_{\varepsilon}\left(\lambda, 2^{\nu}\right)$ for $\nu \leq 26$, we verified this relation by summing up the counts $d_{\varepsilon}\left(\lambda, 2^{\nu}\right)$ derived from Tables 1 and 2 . The counts $d_{\varepsilon}\left(\lambda, 2^{\nu}\right)$ can also be used to compute the number of chains of minimal length $l$ in the range $a_{1}<2^{\nu}$ by evaluating the sum $\sum_{\lambda \geq l} d_{\varepsilon}\left(\lambda, 2^{\nu}\right)$. For example, with $l=2$ and $\varepsilon=+1$ the total number of Sophie Germain primes below $2^{\nu}$ is obtained. For $\nu=20,23,26$ the actual counts are found in Table 6.

In [12] Sumiyama gave an empirical formula for the distribution of prime chains with $\varepsilon=+1$, but only considering chains for which $a_{1} \equiv-1(\bmod 30)$. This congruence is satisfied for all chains with $\lambda \geq 4$ with the exception of $S_{+1}(5,2)$. For the sake of comparison, he counted all chains in question with $a_{1}<3 \cdot 10^{7} \approx 1.79 \cdot 2^{24}$. In our notation, he computed $d_{+1}\left(\lambda, 3 \cdot 10^{7}\right)$ for $\lambda \geq 6$, and $d_{+1}\left(\lambda, 3 \cdot 10^{7}\right)-1$ for $\lambda=4,5$, the reduction by 1 being caused by the chain $S_{+1}(5,2)$. Owing to the congruence relation, for $\lambda \leq 3$ Sumiyama's counts are not comparable with ours. Nevertheless, in spite of the different ways of counting and the deviating range limits, we could confirm all his counts.

3. Approximations. As we have said, it is not known whether arbitrarily long chains exist. It is also not known whether infinitely many chains of a given minimal length $l$ exist, even for $l=2$. However, for any minimal length $l$ and choice of $\varepsilon= \pm 1$, the prime $k$-tuples conjecture [10, Hypothèse $\mathrm{H}$ ] does imply that infinitely many chains of this length and type exist. A quantitative form of this conjecture proposed by Bateman and Horn [1] even predicts how many chains should appear, asymptotically, below a limit $N$.

Specifically, suppose $f_{1}, f_{2}, \ldots, f_{k}$ are linear polynomials in one variable with all coefficients integral and leading coefficients positive, and no two of them differ by a constant factor. Let $Q(N)$ be the number of integers $n, 1 \leq n \leq N$, such that $f_{1}(n), f_{2}(n), \ldots, f_{k}(n)$ are all primes. Then, for large $N$ it is expected that

$$
Q(N) \sim C\left(f_{1}, f_{2}, \ldots, f_{k}\right) \int_{2}^{N} \frac{d u}{(\log u)^{k}}
$$

where

$$
C\left(f_{1}, f_{2}, \ldots, f_{k}\right)=\prod_{j=1}^{\infty}\left\{\left(1-\frac{1}{q_{j}}\right)^{-k}\left(1-\frac{\omega\left(q_{j}\right)}{q_{j}}\right)\right\} .
$$

Here $\omega\left(q_{j}\right)$ denotes the number of distinct solutions of the congruence

$$
f_{1}(x) f_{2}(x) \cdots f_{k}(x) \equiv 0 \quad\left(\bmod q_{j}\right),
$$

$q_{j}$ being the $j$ th prime. It is agreed to exclude the trivial case that $\omega\left(q_{j}\right)=q_{j}$ for some $j$, which would imply $C\left(f_{1}, f_{2}, \ldots, f_{k}\right)=0$.

In the case of prime chains of minimal length $l$ the numbers $\omega\left(q_{j}\right)$ are precisely given by $\min \left(r_{j}, l\right)$, the expression introduced above when our sieving procedure was described. 
On the other hand, for ordinary twin pairs, triplets, and quadruplets of primes, the Bateman-Horn formula reproduces the well-known prediction of Hardy and Littlewood [4, p. 61], which states that for primes $p \leq N$ the number of twins $(p, p+2)$, of triplets $(p, p+2, p+6)$ or $(p, p+4, p+6)$, and of quadruplets $(p, p+2, p+6, p+8)$ asymptotically equals

$$
1.320323632 \int_{2}^{N} \frac{d u}{(\log u)^{2}}, \quad 2.858248596 \int_{2}^{N} \frac{d u}{(\log u)^{3}}, \quad 4.151180864 \int_{2}^{N} \frac{d u}{(\log u)^{4}},
$$

respectively. The constants preceding the integrals are taken from $[9$, p. 65].

When prime chains of minimal length $l=2,3,4$ are considered, the same values of $\omega\left(q_{j}\right)$ as for the respective $l$-tuples are obtained, with only one exception. In the case of chains with $l=4$ we have $\omega\left(q_{4}\right)=3$, while for quadruplets $\omega\left(q_{4}\right)=4$. Thus the contribution of the factor $\left(1-\omega\left(q_{4}\right) / q_{4}\right)$ to the constant $C\left(f_{1}, f_{2}, f_{3}, f_{4}\right)$ is $4 / 7$ for chains, instead of $3 / 7$ for quadruplets. Summarizing, the approximating functions for chains with $l=2,3$ are the same as those given above for twins and triplets, and the number of chains with $l=4$ is approximated by the third function, multiplied by $4 / 3$. To evaluate the integrals we shall choose $N=2^{\nu}$ for certain values of $\nu$, as we did for the limits of all our counts.

In Table 6 we compare the predicted quantities with the actual counts $\sum_{\lambda \geq l} d_{\varepsilon}\left(\lambda, 2^{\nu}\right)$ of chains of minimal length $l=2,3,4$ for $\nu=20,23,26$ and both choices of $\varepsilon$. For $l=4, \nu=29$ is also included. The ratios between the counted and the estimated quantities reflect the behavior of the approximating functions. As the conjecture predicts, for a given $l$ the ratio in question appears to approach 1 for increasing $\nu$. However, for longer chains this convergence becomes noticeably slower. As a matter of fact, meaningful estimates can only be expected if $l$ is small and $\nu$ is sufficiently large. Otherwise, the computed approximation will not even produce the correct order of magnitude. Therefore, it appears to be illusive to use the Bateman-Horn formula to guess where a first chain of length $\lambda=14$ might occur.

Acknowledgments. The author is pleased to thank Wilfrid Keller for his lively interest shown during the preparation of this paper, and especially for having conceived and elaborated the essential part of Section 3. Thanks are also extended to the referee, who kindly pointed out the appropriateness of such considerations.

Rechenzentrum der Universität Hamburg

Hamburg, Federal Republic of Germany

1. Paul T. Bateman \& Roger A. Horn, "A heuristic asymptotic formula concerning the distribution of prime numbers," Math. Comp., v. 16, 1962, pp. 363-367. MR 26 \#6139.

2. Allan Cunningham, "On hyper-even numbers and on Fermat's numbers," Proc. London Math. Soc. (2), v. 5, 1907, pp. 237-274.

3. RiChARD K. GUY, Unsolved Problems in Number Theory, Springer-Verlag, New York, 1981. Updated Japanese edition, Tokyo, 1983.

4. G. H. HARDY \& J. E. LITTLEWOOD, "Some problems of 'partitio numerorum'; III: On the expression of a number as a sum of primes," Acta Math., v. 44, 1923, pp. 1-70.

5. Claude lalout \& Jean Meeus, "Nearly-doubled primes," J. Recreational Math., v. 13, 1980-81, pp. 30-35. 
6. D. H. LEHMER, "On certain chains of primes," Proc. London Math. Soc. (3), v. 14A, 1965, pp. 183-1.86. MR 31 \#2222.

7. R. P. Nederpelt, R. B. Eggleton \& John H. Loxton,. "Problem E 2648," Amer. Math. Monthly, v. 84, 1977, p. 294. Stanley Wagon, Milton Eisner et AL., "Solution and discussion of Problem E 2648," Amer. Math. Monthly, v. 84, 1977, pp. 595-596

8. Paulo Ribenboim, 19 Lectures on Fermat's Last Theorem, Springer-Verlag, New York, 1979.

9. HANS RIESEL, Prime Numbers and Computer Methods for Factorization, Birkhäuser, Boston, 1985.

10. A. SCHINZEL \& W. SIERPIŃSKI, "Sur certaines hypothèses concernant les nombres premiers," Acta Arith., v. 4, 1958, pp. 185-208. MR 21 \#4936.

11. TAKao SumiYama, "Cunningham chains of length 8 and 9," Abstracts Amer. Math. Soc., v. 4, 1983, p. 192, 83T-05-72.

12. TakaO SumiYama, "The distribution of Cunningham chains," Abstracts Amer. Math. Soc., v. 4,1983, p. $489,83 \mathrm{~T}-10-405$.

13. Samuel Yates, Repunits and Repetends, Star Publishing Co., Delray Beach, Florida, 1982. 